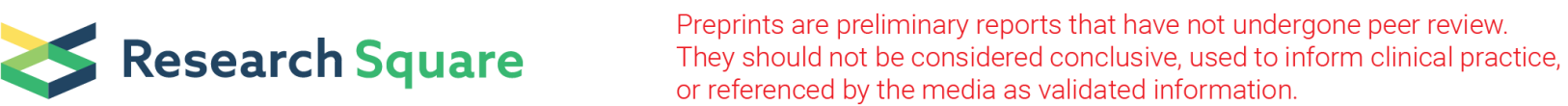

\section{Integrated longitudinal analysis of glioblastomas with long-term relapse interval revealed upregulation of the Tumor Growth Factor $\beta$ signaling pathway in the recurrent tumor}

Elham Kashani

Institute of Pathology, University of Bern, Bern

Désirée Schnidrig

Department for BioMedical Research, University of Bern, Bern

Ali Hashemi Gheinani

Urological Diseases Research Center, Boston Children's Hospital, Boston, MA

Martina Selina Ninck

Institute of Pathology, University of Bern, Bern

Philipp Zens

Institute of Pathology, University of Bern, Bern

Theoni Maragkou

Institute of Pathology, University of Bern, Bern

Ulrich Baumgartner

Cell and Molecular Biology Department, QIMR Berghofer Medical Research Institute, Sid Faithfull Brain

Cancer Laboratory, Brisbane

Philippe Schucht

Department of Neurosurgery, University Hospital Bern, Bern

\section{Gunnar Rätsch}

Department of Computer Science, ETH Zurich, Zurich

Mark Andrew Rubin

Department for BioMedical Research, University of Bern, Bern

Sabina Berezowska

Institute of Pathology, University of Bern, Bern

Charlotte K. Y. Ng

Department for BioMedical Research, University of Bern, Bern

Erik Vassella ( $\nabla$ erik.vassella@pathology.unibe.ch )

Institute of Pathology, University of Bern, Bern 
Keywords: Recurrent glioblastoma, longitudinal analysis, tumor evolution, TGF- $\beta$ signaling, miRNA

Posted Date: February 25th, 2022

DOI: https://doi.org/10.21203/rs.3.rs-1350734/v1

License: (c) (1) This work is licensed under a Creative Commons Attribution 4.0 International License. Read Full License

Version of Record: A version of this preprint was published at Neuro-Oncology on September 17th, 2022. See the published version at https://doi.org/10.1093/neuonc/noac220. 


\section{Abstract}

Background. Glioblastoma (GBM) is the most aggressive primary brain tumor and represents a particular challenge of therapeutic intervention.

Methods. In a single-center retrospective study of matched pairs of initial and post-therapeutic GBM cases with a recurrence period greater than one year, we performed whole exome sequencing combined with mRNA and microRNA expression profiling to identify processes that are altered in recurrent GBM.

Results. Expression and mutational profiling of recurrent GBM revealed evidence for early branching evolution in seventy-five percent of patients. SBS1 signature was reduced in the recurrent tumor and SBS11 was elevated, demonstrating the effect of alkylating agent therapy on the mutational landscape. There was no evidence for secondary genomic alterations driving therapy resistance. ALK7/ACVR1C and $\angle T B P 1$ were upregulated, whereas $L E F T Y 2$ was downregulated, pointing towards enhanced Tumor Growth Factor $\beta$ (TGF- $\beta$ ) signaling in the recurrent GBM. Consistently, altered microRNA expression profiles pointed towards enhanced Nuclear Factor Kappa B signaling that, cooperatively with TGF- $\beta$, induces epithelial to mesenchymal transition (EMT), migration and stemness. In contrast, TGF- $\beta$-induced expression of pro-apoptotic proteins and repression of anti-apoptotic proteins were uncoupled in the recurrent tumor.

Conclusions. Our results suggest an important role of TGF- $\beta$ signaling in recurrent GBM. This may have clinical implication, since TGF- $\beta$ inhibitors have entered clinical phase studies and may potentially be used in combination therapy to interfere with chemoradiation resistance. Recurrent GBM show high incidence of early branching evolution. High tumor plasticity is confirmed at the level of microRNA and mRNA expression profiles.

\section{Background}

Glioblastoma (GBM, WHO grade IV) is the most frequent and aggressive primary brain tumor in adult patients [1]. It is encountered either as the converging end point of progression from a lower-grade glioma (astrocytoma, WHO grade 4, IDH-mutant) or as a full-blown malignant neoplasm at first presentation (de novo GBM, IDH wildtype (wt)). GBM is characterized by extensive plasticity and considerable heterogeneity [1]. Comprehensive analysis of somatic alterations and expression profiling allowed GBM classification into classical (EGFR copy number gains and EGFRvIl/variants), mesenchymal (NF1 alterations, tumor necrosis factor super family pathway and inflammatory infiltrates) and proneural (IDH1 mutations and PDGFRA amplification) subtypes, which often coexist in the same tumor [2]. Although differences depending on the major subtype exist, generally the prognosis of GBM patients is very poor.

Treatment options for GBM patients have not changed over the last 15 years, and the success of targeted therapy or immunotherapy has been limited. Thus, the standard of care for GBM patients remains surgical resection followed by concomitant DNA alkylating agent therapy with temozolomide (TMZ) and radiation therapy (RT) [3]. However, the time to relapse is generally short (6.9 months on average [4]) 
owing to the tumor's ability to infiltrate normal parenchyma and its intrinsic resistance to radiochemotherapy. Due to the lack of alternative treatment options, elucidating underlying resistance mechanism has come into major focus of research, with the hope for opening new ways of therapeutic intervention overcoming resistance.

TMZ induces DNA adducts such as $0^{6}$-methylguanine, which forms mispairing with thymine leading to futile cycles of mismatch repair (MMR) response [5]. DNA double-strand breaks eventually occur in response to this erroneous repair process, which, if not repaired, lead to cytotoxicity. Consequently, defects in MMR enzymes are recognized as major mechanisms of TMZ resistance [6]. The DNA repair enzyme, $0^{6}$-methylguanine DNA methyltransferase (MGMT), removes these adducts from guanine residues and thereby reverses DNA damage induced by alkylating therapy. Indeed, epigenetic silencing of MGMT, which occurs in about $40 \%$ of GBM patients, is associated with a better prognosis in adult GBM patients who received TMZ/RT [7]. Longitudinal analysis of recurrent GBM revealed that driver mutations in the therapy-naïve (hereafter referred to as "initial") tumor were also retained in the post-therapeutic (hereafter referred to as "recurrent") tumor, and there was no evidence for specific secondary alterations driving tumor recurrence [8]. Instead, tumor progression was mainly a result of a highly branched evolutionary process that involved clonal expansion, genetic diversification and clonal selection [8-11]. A trend for proneural to mesenchymal subtype switch during recurrence was previously observed [12], but it is not clear if this was related to treatment resistance. Drivers of the proneural subtype were identified as early events, since they are shared by most subclones, whereas drivers of other subtypes often occurred at a later stage of recurrence [10]. The extensive GBM plasticity as the basis for TMZ resistance is further corroborated by a high epigenetic plasticity in recurrent tumors [13]. MicroRNAs (miRNAs), short regulatory non-coding RNAs that control gene expression at the posttranscriptional level, add additional complexity to the gene regulatory networks [14]. Despite miRNA deregulation being a potential TMZ resistance mechanism [15], there are no reports assessing miRNA profiles of recurrent GBM in a systematic manner.

To explore therapy resistance driving events, we performed a study using a single-centric collective of matched pairs of therapy-naïve and post-therapeutic, formalin-fixed paraffın embedded (FFPE) tissue from 43 GBM patients, selected based on a maximally long interval between resections of the initial and the recurrent tumors. This rare collective was analyzed by whole exome sequencing (WES), mRNA expression and miRNA expression profiling. Here we provide evidence for a high degree of early branching (ancestral) evolution. In addition, we show that deregulated mRNAs and miRNAs in recurrent GBM point towards enhanced tumor necrosis factor- $\alpha$ (TNF- $\alpha$ ) and transforming growth factor- $\beta$ (TGF- $\beta$ ) signaling. To the best of our knowledge, this is the largest single-center retrospective study of matched GBM cases with an exceptionally long recurrence period that were analyzed by multi-omics approaches.

\section{Methods}

\section{Patient cohort}


We included glioblastoma patients with a first surgery at diagnosis and at least one additional surgery upon recurrence following standard treatment, diagnosed at the Institute of Pathology, University of Bern 1999-2016. All tumors were resected at the Department of Neurosurgery, Inselspital University Hospital Bern. The cohort was retrospectively assembled according to the pathology and clinical files. We performed a central review of all cases according to current guidelines (WHO 2021 [1]). IDH mutation status [16], loss of heterozygosity 1p/19q [17], MGMT methylation status [18], and immunohistochemical staining for MIB1 were assessed during routine diagnostic evaluation. Other criteria including H3 mutations, EGFR gene amplification, $+7 /-10$ chromosome copy number changes were assessed by WES (see below). The initial cohort comprised 97 patients with matched FFPE samples from the initial and recurrent tumor. A sub-cohort comprising 43 patients, with an interval of at least one year between pretherapeutic and post-therapeutic resections was selected for molecular analysis. Patient and clinical data of this sub-cohort are shown in suppl. Table 1. All tumors had sufficient tumor purity $(>50 \%)$ by histological analysis.

\section{Tissue microarray and immunohistochemistry}

A tissue microarray (ngTMA) was constructed from at least three punches from the tumor center and, if available, from adjacent normal tissue. Immunohistochemical analysis using monoclonal antibodies directed against ACVR1C/ALK7 (clone AB_1078134, 1:50 dilution), and BCL2 (clone 124, 1:30 dilution) was performed by an automated Leica Bond RX System (Leica Biosystems, Newcastle, UK). Antigen retrieval was performed with Tris- $\mathrm{HCl}, \mathrm{pH} 9$ for $30 \mathrm{~min}$ at $95^{\circ}$. ACVR1C/ALK7 was semi quantitatively evaluated by an experienced neuropathologist (TM) estimating the proportion of positive cells in at least three TMA punches and classifying the cases in: negative (0\%), $0-5 \%, 5-25 \%, 25-50 \%,>50 \%$, positive (100\%). Bcl-2 expression was quantitatively evaluated using QuPath [19]. Initially, nuclei were detected using the StarDist2D model for nucleus segmentation [20]. The detections were further split up in a central, nuclear membrane and cytoplasm compartment and positive cells were identified by applying a threshold of mean DAB (3, 3'-diaminobenzidine) intensity in the nuclear membrane compartment (suppl. Figure 1).

\section{Nucleic acid extraction}

Genomic DNA was extracted from FFPE punches using QIAamp DNA Mini kit (QIAGEN) and analysed using the Agilent NGS FFPE QC kit. $93 \%$ of samples showed sufficient DNA quality as indicated by a $\triangle \mathrm{Ct}$ value $<2$. Total RNA was extracted using the RecoverAll Total Nucleic Acid Isolation Kit for FFPE (ThermoFisher). For miRNA profiling, total RNA was purified using the Zymo Research DNA Clean and Concentrator-5 kit (Zymo Research, Irvine, CA, USA).

\section{Library preparation and whole-exome sequencing}

Libraries were prepared using the SureSelectXT Human All Exon V7 Low Input Reagent Kit and SureSelect XT Low Input P5 indexed adaptors (Agilent). Sequencing was performed on a NovaSeq 6000 using the S2 Reagent Kit (200 cycles). The sequencing depth was in a range of 25-130 (median 77).

\section{Processing of WES data}


Processing and somatic variant calling of the WES data was performed using the T/N workflow from Bcbio-nextgen v1.1.7 [21]. Gene-level copy number alterations were determined with Sequenza v3.0.0 [22] and GISTIC v2.0.23 [23]. See also Supplementary Methods.

\section{Mutational signatures and TMB}

Mutational signatures were inferred with MutationalPatterns v3.0.0 [24] and deconstructSigs v1.8.0 [25], for the COSMIC signatures v3 (May 2019) previously described in the GLASS [8] or PCWAG CNS-GBM cohort [26] [SBS1, SBS3, SBS5, SBS8, SBS11, SBS15, SBS16, SBS30, SBS40]. Paired Wilcoxon tests were used to compare signature contributions and TMB values in 'initial' and 'recurrent' samples. See also Supplementary Methods.

\section{Subclonal and phylogenetic reconstruction and evolutionary league model}

Tumor sample purity, average ploidy and cancer cell fractions of mutations were estimated with ABSOLUTE v1.2 [27] in allelic mode. Phylogenetic reconstruction and evolutionary league model inference were performed with PhylogicNDT [28, 29]. See also Supplementary Methods.

\section{Nanostring expression profiling of mRNAs and miRNAs}

One hundred ng total RNA was analysed using the nCounter Human miRNA Expression Panel Assay Kit H_miRNA_V3 or the nCounter Human PanCancer pathway panel (NanoString, Seattle, WA, USA) spiked with 30 additional genes implicated in autophagy, EMT and DNA repair processes (suppl. Table 2), as previously described [30]. See Supplementary Methods for bioinformatics analysis.

Due to large dynamic expression range (suppl. Figure 2A,C), negative binominal distribution model was chosen as the normalization method for mRNA and miRNA data to adjust for data overdispersion (variance > mean), i.e. heteroscedasticity, and to avoid false-positive discoveries. The distribution of raw mRNA and miRNA counts were comparable across all samples (suppl. Figure 2B,D). A similar dispersion of the variance from mean of counts was observed for mRNAs (suppl. Figure 2A) and miRNAs (suppl. Figure 2C) from initial, recurrent and normal tissue. No bias for gender was noted based on the distribution of raw non-normalized Nanostring counts for two randomly nominated genes (B2M and TNF) (suppl. Figure 2E).

\section{Calculation of Hazard Ratio in publicly available datasets}

For assessment of the prognostic importance of differentially expressed genes, we used the TCGA glioblastoma cohort including a total of 592 patients with RNA-Seq data available for 160 samples [31]. The datasets were downloaded on 27.01.2022 and clinical data as well as z-score normalized RNA-Seq data (based on all samples) was used. Univariable cox proportional hazard models were used to estimate the effect of gene expression and a p-value $<0.05$ was deemed to be statistically significant. 
TCGA data analysis was performed using TCGAbiolinks R package [32]. Normalized counts (Fragments Per Kilo base of transcript per Million mapped reads [FPKM]) and Normalized HTSeq counts were used for statistics. A univariable cox proportional model for common GBM driver genes (IDH1, PTEN, PIK3R1, $T P 53$, etc.) was evaluated. Genes achieving a significance level of $p<0.1$ were included in a multivariable model. Maximally selected rank statistics was utilized to find the optimal cut-off for gene expression dichotomization. Hazard ratio was calculated using multivariable cox-regression statistical model.

\section{In vitro validation of ALK7 modulation in a GBM cell line}

Authenticated U87MG cells were cultured as previously described [33]. 150 hundred U87MG cells were seeded in a 6-well plate 16 hours prior to transfection using the "Single piggyBac vector" system described by Michael et.al [34], except that TransIT-LT1 Transfection Reagent was used (Mirus Bio, USA) to minimize toxicity on U87 cells. Cells were treated with TMZ or DMSO [33] 48 hours post Doxycycline induction. Respective wells were imaged 48 hours post TMZ treatment in at least 3 field-of-views. Apoptotic cell counts were determined after counting cells from 3 randomly selected fields per each condition (approximately 150 cells).

\section{Results}

\section{Sample characteristics}

Our initial cohort comprised 97 patients for which tissues were available from resections at diagnosis (initial GBM) and at recurrence following standard treatment. The time interval between both resections was less than one year for 54 patients and 370-1890 (median 689) days for the remaining 43 patients (suppl. Figure 3A and suppl. Table 1). Among those patients with long-term recurrence (who were included in downstream molecular analysis), 39 had $I D H$ wild-type and four had $I D H$ mutation. The patient age of the subcohort with long-term recurrence ranged from 32-79 (median 57.5) years at initial resection (suppl. Figure 3B). Fourteen patients had frontal, five patients occipital, eleven patients parietal and thirteen patients temporal location of the tumor (suppl. Figure $3 \mathrm{C}$ ). All patients showed recurrence at the original tumor location. 42 patients received concomitant alkylating agent therapy with TMZ and RT and one patient received radiotherapy only. The MIB1 proliferation index was generally lower in recurrent tumors compared to the initial tumor (suppl. Figure 3D). In agreement with published results, no significant difference in the MGMT methylation status was noted between initial and recurrent tumors (suppl. Figure 3E). Of note, the proportion of tumors with hypermethylated MGMT promoter at diagnosis was significantly higher in patients with a recurrence interval greater than one year compared to patients with a shorter recurrence interval $(p=0.03)$ [7].

\section{Mutational burden and mutational signatures in the recurrent tumor of patients with long-term relapse interval}

WES was performed using trios of matched initial, recurrent GBM and normal tissue from 28 patients with long-term relapse interval. All these patients had received TMZ and concomitant RT. 25 patients had 
GBM IDH wt and 3 patients had GBM IDH mutant. We observed a range of 0.7-46.0 (median 1.6) mutations per megabase (mut/Mb) for the initial and a significantly higher range of 1.1-66.8 (median 2.4) mut/Mb for the recurrent tumors (Fig. 1A). Most samples with high TMB revealed a high rate of SBS11 mutation signature (TMZ treatment) (Fig. 1B,C). Although post-therapeutic mutations in MMR genes were detected in 7/28 patients, they were not associated with SBS15 (defective MMR), the latter being present only at a very low rate (suppl. Figure $4 A \& B$, suppl. Table 3 ). Instead, mutations in MMR genes were mainly present in tumors with high SBS11 signature (suppl. Figure 4B), suggesting that the high mutational burden was most likely caused by the hypermutator activity of TMZ rather than defects in MMR genes. TMB was not associated with the age of the patient at initial excision (suppl. Figure 4C).

We also found a significant decrease in the contribution of the SBS1 signature (deamination of $5 \mathrm{MeC}$ ) in recurrent tumors (Fig. 1C), which may in part be secondary to the strong enrichment of the SBS11 (TMZ signature) in some recurrent tumors. This observation is concordant with GLASS [8]. There was no correlation between the extent of SBS1 signature and the age of the paraffin block indicating that the SBS1 signature was not caused by pre-analytical oxidation of the DNA (data not shown), a phenomenon described in old paraffin blocks. The frequency of signatures associated with defects in different DNA repair systems was not altered between initial and recurrent tumors (suppl. Figure 4A).

\section{Mutational landscape of tumors with late recurrence}

Most tumors showed a copy number gain at chromosome 7 and a copy number loss at chromosome 10, which is characteristic for GBM IDH wt (Fig. 2 and suppl. Figure 5A\&B and suppl. Figure 6), except GBM $\# 26,73,82$ and 88 , which showed no copy number gain at chromosome 7 and \#86, which showed no copy number loss at chromosome 10. Interestingly, an almost identical Copy Number Variation (CNV) profile was retained in the recurrent tumor in the majority of cases (suppl. Figure $5 A, B$ ). Likewise, known drivers of GBM occurring in the initial tumor including IDH1 (2/2), PTEN (5/7), PIK3CA (4/6), PIK3R1 (3/3) and TP53 (5/5) mutations were often retained in the recurrent tumor. In contrast, mutations in EGFR (9/11), NF1 (7/10), RB1 (3/4) and amplifications of EGFR (4/8), PDGFRA (3/3) and MDM2 (2/3) were either private to the initial or recurrent tumor (Fig. 2 and suppl. Table 3). These subclonal alterations are characteristic for either classical, proneural or mesenchymal subtypes, indicating a high plasticity of GBM during recurrence. The prevalence of these alterations in initial tumors in our dataset was similar to those of the TCGA dataset, which comprises mainly therapy-naïve, initial tumors with shorter recurrence. The only exception was NF1 mutations, which were more abundant in our dataset (5/27 initial tumors: $19 \%$ ) compared to the TCGA dataset (9.3\%) (data not shown). Mutations that were most frequently found to be private to recurrent tumors, were also enriched in cases with high TMB, and there was no evidence for recurrent driver alterations (suppl. Figure 7 and suppl. Table 3).

\section{Tumor evolution of recurrent GBM with long-term therapy response}


In a next step, the clonal architecture during progression and recurrence of the tumor was analyzed. Across the cohort, PTEN mutations were early events whereas potentially targetable alterations in EGFR, mTOR and NF1 appeared later during tumor development (Fig. 3A). Longitudinal analysis revealed a persistent mutational cluster in only 5/20 (25\%) of patients (Fig. 3C). Instead, in 15/20 (75\%) cases, the dominant subclone in the recurrent tumor was a direct linear descendant of the founding clone, suggesting early branching as the predominant pattern of evolution (Fig. 3B and suppl. Figure 8). A similar finding was obtained by Wang et al [35] who reported that the dominant subclone of the initial tumor was absent in the recurrence in $59 \%$ of cases. This class of evolution is termed ancestral evolution [36], and was described by Kim et al [10]. Our finding of a high proportion of GBM with early branching evolution may have clinical implications. For example, patient \#052 carries an actionable EGFR p.A289V mutation, which is present in the major subclone of the initial tumor (suppl. Figure 8). However, the EGFR inhibitor lapatinib would not be a suitable secondary therapeutic option for this patient, since this mutation is absent in the recurrent tumor. In contrast, patient \#086, who carries a truncal BRAF p.V600E mutation, more likely responds to a secondary therapy with BRAF inhibitors (suppl. Figure 8).

\section{TNF and TGF- $\beta$ signalling pathways are enhanced in the recurrent tumor}

Longitudinal mRNA and miRNA expression analysis was performed from matched initial and recurrent GBM tissues of 43 patients, and normal tissues adjacent to the initial tumors from 10 patients in order to identify pathways that are altered in recurrent GBM. To this end, we used Nanostring technology, which relies on hybridization and therefore gives rise to a high correlation in the expression profile of RNA from FFPE and fresh-frozen tissues [37]. The pan cancer panel (800 genes), which comprises genes implicated in proliferation, angiogenesis, cancer metabolism, epithelial to mesenchymal transition (EMT), metabolism and DNA repair was spiked with 30 additional genes implicated in TMZ resistance (suppl. Table 2). We performed dimension reduction hierarchical clustering and sample-level QC using Principal Component Analysis (PCA) with normalized counts to investigate similarities between samples. As expected, mRNA and miRNA expression patterns of samples derived from normal adjacent brain tissue were clustered separately from those derived from tumor tissues (Fig. 4A and suppl. Figure 9A,B). However, neither initial-recurrent tumor groups nor individual patient pairs were clustered together, confirming high plasticity of recurrent GBM at the transcript level. Consistent with this finding, EGFR expression, a marker of the classical subtype, was strongly upregulated in the recurrent tumor of some patients, but strongly downregulated in the recurrent tumor of other patients (Suppl. Figure 10).

Differential gene expression analysis of initial and recurrent tumor pairs indicated significant deregulation of multiple genes (suppl. Table 4). The top ranked genes were ALK7 (ACVR1C), RASAL1, COMP, LTBP1, HDAC11 and BCL2, which were upregulated, and MDM2 and LEFTY2, which were downregulated in recurrent tumors with significant adjusted p-value (Fig. 4B\&C). Notably, with the exception of COMP, all genes were dysregulated in multiple patients as indicated by circos plot (suppl. Figure 11) suggesting a common, gender-neutral mechanism in recurrent tumors. The differentially 
expressed genes were used for survival analysis from TCGA data. In univariable analyses, only LTBP1 ( $\mathrm{p}$ $=0.027$ ) was significantly associated with worse survival (suppl. Figure 12). MiR-542-5p, miR-574-3p, miR-129-3p, miR-148a-3p, miR199b-5p, miR-129-5p, miR-196a-5p, miR-219a-5p, miR-199a-5p were upregulated and miR-1246, miR-494-3p, miR-19b-3p and miR-630 were downregulated in recurrent GBM (Fig. 4B\&C). Interestingly, miR-129-5p, miR-1246, miR-494-3p and miR-19b-3p have been linked to TMZ resistance by targeting WNT signaling [38], CCNG2 [39], p-AKT [40] and PTEN [41], respectively.

Gene set enrichment analysis for GO, KEGG and Reactome of differentially expressed mRNAs revealed a significant enrichment of transmembrane protein kinase activity (NES $=+1.5)$, TGF- $\beta$ signaling (NES $=+$ 1.3) and downregulation of $p 53$-independent G1/S DNA damage checkpoints (NES=-1.5) among others (Fig. 5A, upper). Biological function analysis based on regulated pathways for differentially expressed mRNAs revealed a significant regulation of disease-specific pathways, cellular immune response, cellular growth, proliferation, development, and apoptosis, consistent with increased TMZ resistance (Fig. 5B,upper). Gene network analysis allowed the identification of potential master regulators of differentially regulated mRNAs leading to enhanced phosphorylation (Suppl. Figure 13). Interestingly, differentially regulated mRNAs and predicted targets of differentially expressed miRNA revealed an almost complete overlap of biological functions (Fig. 5B, lower), although different pathways were induced (Fig. 5A, lower), suggesting that mRNAs and miRNAs act in a concerted manner. Integrated analysis of dysregulated miRNAs and their inversely correlating miRNA targets allowed the identification of miRNA/mRNA negative regulation pairs, with miR-19b-3p predicted to be most frequently engaged in binding to dysregulated mRNAs (Fig. 5C\&D). TNF was the highest induced transcript (log ratio $=+1.051$ ) in recurrent tumors by the miRNA/mRNA negative regulation pairs (mainly due to the miR-19b-3p downregulation) (Fig. 5E). Accordingly, biological function and integration analyses based on differentially regulated mRNAs, differentially regulated miRNAs, and targets oppositely regulated by miRNAs, pointed towards enhanced disease-specific pathways, proliferation and developmental pathways (Fig. 5A,B and suppl. Figure 14).

\section{ALK7 is enhanced in the recurrent tumor}

ALK7, a member of the type I TGF- $\beta$ receptor subfamily implicated in EMT [42], was most significantly upregulated in recurrent GBM (Fig. 4B,C). Pairwise comparison of matched initial and recurrent tumors confirmed ALK7 upregulation (Fig. 6A). To assess ALK7 expression at the protein level, a tissue microarray was constructed from the patient cohort with long-term resection intervals and analysed by immunohistochemistry using an ALK7 antibody. IHC scoring was based on the percentage of ALK7 positive tumor cells at recurrence relative to the initial tumor. ALK7 was indeed upregulated in the majority of recurrent tumor punches with a significant positive correlation $\left(R^{2}=0.2614, p<0.001\right)$ with Nanostring normalized counts (Fig. 6B). Overexpression of a dominant negative form of ALK7, containing a p.K222R kinase dead mutation[34] conferred enhanced TMZ-induced cell death in U87 GBM cells, suggesting that ALK7 overexpression is a resistance mechanism in recurrent GBM (suppl. Figure 15A). In non-neoplastic cells, ALK7 induces apoptosis through upregulation of pro-apoptotic Bax and downregulation of anti- 
apoptotic factors $\mathrm{Bcl}-2, \mathrm{Bcl}-\mathrm{XL}$, and XIAP [42]. Consistent with these findings, overexpression of a constitutively active form of ALK7 containing a p.T194D mutation [34], but not the dead mutant form, strongly induced apoptosis in our GBM cell line model (suppl. Figure 15B). How can glioma cells deal with ALK7 overexpression? ALK7 mRNA expression was directly correlated with $B C L 2$ expression and inversely correlated with $B A K 1$ and $B A X$ expression in the TCGA dataset of initial GBM (suppl.

Figure 16A), suggesting that the apoptosis supporting role of ALK7 is reversed in recurrent GBM. In line with TCGA data, $A L K 7$ and $B A X$ mRNA expression was inversely correlated in our dataset of long-term responders (suppl. Figure 16B). Although BCL2 was significantly upregulated in recurrent GBM (Fig. 4B,C), which was confirmed by pairwise comparison of matched initial and recurrent tissues (Fig. 6C,D and suppl. Figure 1), BCL2 and $A L K 7$ were not significantly correlated in our dataset (data not shown).

\section{Discussion}

We performed a comprehensive analysis of a single-center cohort of patients with matched initial and recurrent tumors resected at a time interval greater than one year. We expected to gain a better insight into the molecular mechanisms underlying resistance from this unique cohort of long-term responders. We provide evidence for a highly branched evolution of recurrent GBM with a high proportion of patients showing early divergent (ancestral) evolution defined by the outgrowth of distinct subclones in the initial and recurrent tumors, which have been branched from a common ancestor long before diagnosis [10]. This type of evolution results in the disappearance of the initial clone(s) following therapy and the outgrowth of a new clone from an ancestor cell, which shares only truncal mutations with the initial clone. In contrast, patient collectives from other studies, which also include short-term survivors, tend to show dominance of clones in the initial tumor that progress during recurrence [8]. It is conceivable that clones from tumors with a short-term recurrence interval show higher intrinsic resistance to standard therapy. Highly branched clonal evolution of GBM at the mutational level is further corroborated by the finding that high plasticity also exists for mRNA and miRNA profiles (our data), as well as epigenetic profiles [13]. This may have clinical implications, since potentially actionable alterations, such as those in EGFR, NF1 or MTOR, may dominate the initial tumor, but may disappear during recurrence rendering targeted therapy ineffective.

In agreement with previous studies [8], there was no evidence for recurring secondary alterations at genomic level driving TMZ/RT resistance. Instead, known drivers of GBM were often private to either the initial or the recurrent tumor. Some of these alterations are drivers of proneural, classical or mesenchymal subtypes [9], but we noted no significant enrichment for a certain subtype, which is in line with previous findings [10,43]. TP53 mutations appeared early during tumor evolution in line with previous findings [10], but a discrepancy exists for PTEN mutations, which was an early event in our study and a late event in the study by Kim et al [10]. Whether this is due to a bias for late recurrence in our patient cohort remains to be shown. In contrast to a high plasticity for mutations, the CNV profile remained largely unchanged during progression. The new WHO classification [1], recommends consulting CNV profiles for the classification of GBM. Thus, our finding, that CNV profiles are retained in recurrent tumors even in 
those GBM cases with ancestral evolution, is important in the context of the new GBM classification guidelines, since they also apply to the recurrent tumor.

It was previously shown that TMZ treatment is associated with a significant increase in the mutational burden, which was also confirmed by our study. We found in our cohort of long-term responders that SBS11 (TMZ treatment) and SBS1 (deamination of 5-methylcytosine) were the only mutational signatures that were altered in recurrent GBM. The latter signature is caused by enhanced BER activity in order to repair alkylating bases $\mathrm{N}^{7}-\mathrm{MeG}$ and $\mathrm{N}^{3}-\mathrm{MeA}$, introduced into the DNA by alkylating therapy [44]. However, no evidence for altered SBS15 signature (defective MMR) was obtained.

Messenger RNA expression profiling revealed that $A L K 7$ and $\angle T B P 1$ were significantly upregulated while LEFTY2 was significantly downregulated in recurrent GBM. Most strikingly, all these genes contribute to enhanced TGF- $\beta$ signaling. ALK7 is a brain-enriched type-I-receptor family member of the TGF- $\beta$ superfamily [42], which is induced by Activin B, Activin AB and Nodal [34] and exert its functions by interacting with type II receptors leading to enhanced SMAD2/3 phosphorylation and nuclear translocation, promoting EMT (Fig. 6E) and TMZ resistance (suppl. Figure 15A). LTBP1 encodes a protein binding to TGF- $\beta$ leading to its activation. Another member of the LTBP family, LTBP4, was previously shown to be mutated in $11 \%$ of GBM patients and was always private to the recurrent tumors [10]. We identified $\angle T B P 4$ mutations in $14 \%$ of GBM, and again, all were private to the recurrent tumors. Left-right determination factor LEFTY2 is a member of the transforming growth factor $\beta$ (TGF- $\beta$ ) superfamily. It is activated by the TGF- $\beta$ signaling and confers its repression by a negative feedback loop [45]. Thus, reduced expression of LEFTY2 may result in constitutive TGF- $\beta$ activity, and tumorigenesis (Fig. 6E). The TGF- $\beta$ signal is often reduced during tumor initiation to overcome its anti-proliferative and pro-apoptotic effects but is reactivated during progression to enhance EMT and stemness [42, 45-47]. This dichotomous function results in a non-monotonic expression pattern of normal brain tissue, initial and recurrent tumor, a finding also confirmed in our study. How can recurrent GBM overcome the proapoptotic signal? We show that anti-apoptotic protein BCL2 is induced whereas pro-apoptotic BAX is reduced in recurrent GBM, suggesting that TGF- $\beta$-induced apoptosis is uncoupled in recurrent GBM. It remains to be shown if early branching evolution may support the dichotomous pattern of TGF- $\beta$ signaling since tumor clones with reduced TGF- $\beta$ activity in the initial tumor may be replaced by new clones with enhanced TGF- $\beta$ activity.

Interestingly, dysregulated mRNAs and miRNAs may be implicated in similar cellular processes although they seem to induce different pathways. Thus, enhanced TGF- $\beta$ signaling elicited by dysregulated mRNAs and enhanced TNF-a signaling elicited by dysregulated miRNAs, may cooperatively induce SMAD2 phosphorylation to enhance EMT [46]. RASAL1 and HDAC overexpression has been associated with an unfavourable prognosis of GBM patients. RASAL1 overexpression induces proliferation and migration, and confers enhanced EMT by inducing MEK/ERK signalling [47]. Overexpression of HDAC proteins is associated with radiotherapy resistance[48], but it is unknown if this is due to EMT.

\section{Conclusions}


Combined expression profiling and mutational profiling of recurrent GBM with long-term recurrence provided evidence for early branching evolution in the majority of cases, which may have clinical implications. In addition, our results suggest a major role of the TGF- $\beta$ signaling in recurrent GBM. Thus, administering TGF- $\beta$ inhibitors in conjunction with standard therapy may interfere with TMZ/RT resistance mechanisms.

\section{Abbreviations}

EMT, epithelial to mesenchymal transition, GBM, glioblastoma, miRNA, microRNA, MGMT, $0^{6}$ methylguanine DNA methyltransferase, MMR, mismatch repair, RT, radiotherapy, TMB, tumor mutational burden, TNF, tumor necrosis factor, TGF, tumor growth factor, TMZ, temozolomide.

\section{Declarations}

\section{Ethics approval and consent to participate}

The study was approved by the Cantonal Ethics Commission of the Canton of Bern (KEK-BE: 201800663), which waived the requirement for written informed consent.

\section{Consent for publication}

Not applicable

\section{Availability of data and materials}

The datasets supporting the conclusions of this article is available in the European Genome-Phenotype Archive (EGA) under the accession EGAS00001006022 (for WES Bam files) and is provided the time of submission as additional files (for post-processed WES data and gene expression datasets).

\section{Competing interests}

The authors declare that they have no competing interests.

\section{Funding}

This work was supported by a grant from the Swiss National Science Foundation (grant number 31003A_138129, to E.V.). The SOCIBP (Swiss Molecular Pathology Breakthrough Platform) is a driver project funded by the Swiss Personalized Health Network (SPHN).

\section{Authors' contributions}

E.K., D.S., A.H.G., C.K.Y.N. and E.V. designed the study and the experiments. E.K. performed experiments. E.K., D.S., A.H.G., P.Z., C.K.Y.N. and E.V. performed analysis of the results. E.V. developed the concept and acquired funding. D.S. performed bioinformatic analysis of WES dataset, with contributions from G.R., 
M.A.R. and the SOCIBP consortium. E.K., A.H.G. and U.B. performed bioinformatics analysis of Nanostring data. M.S.N. and S.B. constructed the TMA and performed the pathology review. E.K. and T.M. performed immunohistochemical evaluation. P.Z. performed immunohistochemical confirmation using Digital Pathology. P.S. provided clinical data. E.V. and E.K. wrote the initial draft of the manuscript.

\section{Acknowledgments}

The authors gratefully acknowledge the molecular pathology team of the clinical genomics lab, University hospital Inselspital, and the Translational Research Unit of the Institute of Pathology, University of Bern, for excellent technical support. D. Hanahan, Laboratory of translational oncology, EPFL, generously provided constitutively active and dead mutant ALK7 plasmids.

Group authorship:

SOCIBP consortium (Swiss Molecular Pathology Breakthrough Platform)

Members: Andrej Benjak, Rémy Bruggmann, Federico Comoglio, André Kahles, Irene Keller, Charlotte K Y $\mathrm{Ng}$, Salvatore Piscuoglio, Laurie Prélot, Gunnar Rätsch, Mark A Rubin, Désirée Schnidrig, Senija SelimovicHamza, Tinu Mary Thomas

Affliations: Department for BioMedical Research, University of Bern, Bern, Switzerland (A.B., C.K.Y.N., M.A.R., D.S., S.S.-H.), SIB Swiss Institute of Bioinformatics, Lausanne, Switzerland (A.B., R.B., F.C., A.K., I.K., C.K.Y.N., L.P., G.R., D.S., T.M.T.), Interfaculty Bioinformatics Unit, University of Bern, Bern, Switzerland (R.B., F.C., I.K.), ETH Zurich, Zurich, Switzerland and University Hospital Zurich, Zurich, Switzerland (A.K., L.P., G.R., T.M.T.), Bern Center for Precision Medicine, Bern, Switzerland (C.K.Y.N., M.A.R.), Department of BioMedicine, University of Basel, Basel, Switzerland and Institute of Medical Genetics and Pathology, University Hospital Basel, Basel, Switzerland (S.P.), Inselspital, Bern, Switzerland (M.A.R.).

\section{References}

1. Louis DN, Perry A, Wesseling P, Brat DJ, Cree IA, Figarella-Branger D, et al. The 2021 WHO classification of tumors of the central nervous system: A summary. Neuro. Oncol. 2021,23:1231-51.

2. Neftel C, Laffy J, Filbin MG, Hara T, Shore ME, Rahme GJ, et al. An Integrative Model of Cellular States, Plasticity, and Genetics for Glioblastoma. Cell 2019,178:835-849.e21.

3. Stupp R, Hegi ME, Mason WP, van den Bent MJ, Taphoorn MJ, Janzer RC, et al. Effects of radiotherapy with concomitant and adjuvant temozolomide versus radiotherapy alone on survival in glioblastoma in a randomised phase III study: 5-year analysis of the EORTC-NCIC trial. Lancet Oncol. 2009,10:459-66.

4. Wen PY, Kesari S. Medical Progress Malignant Gliomas in Adults. N Engl J Med 2008,359:492-507.

5. Knizhnik A v., Roos WP, Nikolova T, Quiros S, Tomaszowski KH, Christmann M, et al. Survival and Death Strategies in Glioma Cells: Autophagy, Senescence and Apoptosis Triggered by a Single Type 
of Temozolomide-Induced DNA Damage. PLoS One 2013,8.

6. Johnson BE, Mazor T, Hong C, Barnes M, Aihara K, McLean CY, et al. Mutational analysis reveals the origin and therapy-driven evolution of recurrent glioma. Science 2014,343:189-93.

7. Hegi ME, Diserens A-C, Gorlia T, Hamou M-F, de Tribolet N, Weller M, et al. MGMT Gene Silencing and Benefit from Temozolomide in Glioblastoma. N Engl J Med, 2005,10,352(10):997-1003.

8. Barthel FP, Johnson KC, Varn FS, Moskalik AD, Tanner G, Kocakavuk E, et al. Longitudinal molecular trajectories of diffuse glioma in adults. Nature 2019,576:112-20.

9. Verhaak RGW, Hoadley KA, Purdom E, Wang V, Qi Y, Wilkerson MD, et al. Integrated Genomic Analysis Identifies Clinically Relevant Subtypes of Glioblastoma Characterized by Abnormalities in PDGFRA, IDH1, EGFR, and NF1. Cancer Cell 2010,17:98-110.

10. Kim H, Zheng S, Amini SS, Virk SM, Mikkelsen T, Brat DJ, et al. Whole-genome and multisector exome sequencing of primary and post-treatment glioblastoma reveals patterns of tumor evolution. Genome Res. 2015,25:316-27.

11. Park AK, Kim P, Ballester LY, Esquenazi Y, Zhao Z. Subtype-specific signaling pathways and genomic aberrations associated with prognosis of glioblastoma. Neuro. Oncol. 2019,21:59-70.

12. Bhat KPL, Balasubramaniyan V, Vaillant B, Ezhilarasan R, Hummelink K, Hollingsworth F, et al. Mesenchymal Differentiation Mediated by NF-kB Promotes Radiation Resistance in Glioblastoma. Cancer Cell 2013,24:331-46.

13. Klughammer J, Kiesel B, Roetzer T, Fortelny N, Nemc A, Nenning KH, et al. The DNA methylation landscape of glioblastoma disease progression shows extensive heterogeneity in time and space. Nat. Med. 2018,24:1611-24.

14. Piwecka M, Rolle K, Belter A, Barciszewska AM, Zywicki M, Michalak M, et al. Comprehensive analysis of microRNA expression profile in malignant glioma tissues. Mol. Oncol. 2015,9:1324-40.

15. Banelli B, Carra E, Barbieri F, Würth R, Parodi F, Pattarozzi A, et al. The histone demethylase KDM5A is a key factor for the resistance to temozolomide in glioblastoma. Cell Cycle 2015,14:3418-29.

16. Hewer E, Vajtai I, Dettmer MS, Berezowska S, Vassella E. Combined ATRX/IDH1 immunohistochemistry predicts genotype of oligoastrocytomas. Histopathology 2016,68:272-8.

17. Mariani L, Deiana G, Vassella E, Fathi AR, Murtin C, Arnold M, et al. Loss of heterozygosity $1 p 36$ and $19 q 13$ is a prognostic factor for overall survival in patients with diffuse WHO grade 2 gliomas treated without chemotherapy. J. Clin. Oncol. 2006,24:4758-63.

18. Vassella E, Vajtai I, Bandi N, Arnold M, Kocher V, Mariani L. Primer extension based quantitative polymerase chain reaction reveals consistent differences in the methylation status of the MGMT promoter in diffusely infiltrating gliomas (WHO grade II-IV) of adults. J. Clin. Oncol. 2011,104:293303.

19. Bankhead P, Loughrey MB, Fernández JA, Dombrowski Y, McArt DG, Dunne PD, et al. QuPath: Open source software for digital pathology image analysis. Sci. Reports 201771 2017,7:1-7. 
20. Han L, Yin Z. A Cascaded Refinement GAN for Phase Contrast Microscopy Image Super Resolution. Lect. Notes Comput. Sci. (including Subser. Lect. Notes Artif. Intell. Lect. Notes Bioinformatics) 2018,11071 LNCS:347-55.

21. bcbio. GitHub - bcbio/bcbio-nextgen: Validated, scalable, community developed variant calling, RNAseq and small RNA analysis [Internet]. commit= 38223f89a8d6e20327efa3f094d2a634bd0907bc. [cited 2022 Jan 31],Available from: https://github.com/bcbio/bcbio-nextgen

22. Favero F, Joshi T, Marquard AM, Birkbak NJ, Krzystanek M, Li Q, et al. Sequenza: allele-specific copy number and mutation profiles from tumor sequencing data. Ann. Oncol. Off. J. Eur. Soc. Med. Oncol. 2015,26:64-70.

23. Mermel CH, Schumacher SE, Hill B, Meyerson ML, Beroukhim R, Getz G. GISTIC2.0 facilitates sensitive and confident localization of the targets of focal somatic copy-number alteration in human cancers. Genome Biol. 2011,12:1-14.

24. Blokzijl F, Janssen R, van Boxtel R, Cuppen E. MutationalPatterns: Comprehensive genome-wide analysis of mutational processes. Genome Med. 2018,10:1-11.

25. Rosenthal R, McGranahan N, Herrero J, Taylor BS, Swanton C. DeconstructSigs: delineating mutational processes in single tumors distinguishes DNA repair deficiencies and patterns of carcinoma evolution. Genome Biol. 2016,17.

26. Alexandrov LB, Kim J, Haradhvala NJ, Huang MN, Tian Ng AW, Wu Y, et al. The repertoire of mutational signatures in human cancer. Nature 2020,578:94-101.

27. Carter SL, Cibulskis K, Helman E, McKenna A, Shen H, Zack T, et al. Absolute quantification of somatic DNA alterations in human cancer. Nat. Biotechnol. 2012,30:413-21.

28. broadinstitute. GitHub - broadinstitute/PhylogicNDT [Internet]. commit = 84d3dd2713ed989dbe038fee54fdd0cd564d1a45 [cited 2022 Feb 18],Available from: https://github.com/broadinstitute/PhylogicNDT

29. Leshchiner I, Livitz D, Gainor JF, Rosebrock D, Spiro O, Martinez A, et al. Comprehensive analysis of tumour initiation, spatial and temporal progression under multiple lines of treatment. bioRxiv $2019,508127$.

30. Baumgartner U, Berger F, Hashemi Gheinani A, Burgener SS, Monastyrskaya K, Vassella E. miR-19b enhances proliferation and apoptosis resistance via the EGFR signaling pathway by targeting PP2A and BIM in non-small cell lung cancer. Mol. Cancer 2018,17.

31. Hoadley KA, Yau C, Hinoue T, Wolf DM, Lazar AJ, Drill E, et al. Cell-of-Origin Patterns Dominate the Molecular Classification of 10,000 Tumors from 33 Types of Cancer. Cell 2018,173:291.

32. Colaprico A, Silva TC, Olsen C, Garofano L, Cava C, Garolini D, et al. TCGAbiolinks: An R/Bioconductor package for integrative analysis of TCGA data. Nucleic Acids Res. 2016,44:e71.

33. Haemmig S, Baumgartner U, Glück A, Zbinden S, Tschan MP, Kappeler A, et al. miR-125b controls apoptosis and temozolomide resistance by targeting TNFAIP3 and NKIRAS2 in glioblastomas. Cell Death Dis. 2014,5. 
34. Michael IP, Saghafinia S, Tichet M, Zangger N, Marinoni I, Perren A, et al. ALK7 Signaling Manifests a Homeostatic Tissue Barrier That Is Abrogated during Tumorigenesis and Metastasis. Dev. Cell 2019,49:409-424.e6.

35. Wang J, Cazzato E, Ladewig E, Frattini V, Rosenbloom DIS, Zairis S, et al. Clonal evolution of glioblastoma under therapy. Nat. Genet. 2016487 2016,48:768-76.

36. Aldape K, Amin SB, Ashley DM, Barnholtz-Sloan JS, Bates AJ, Beroukhim R, et al. Glioma through the looking GLASS: Molecular evolution of diffuse gliomas and the Glioma Longitudinal Analysis Consortium. Neuro. Oncol. 2018,20:873-84.

37. Chen X, Deane NG, Lewis KB, Li J, Zhu J, Washington MK, et al. Comparison of Nanostring nCounter ${ }^{\circledR}$ Data on FFPE Colon Cancer Samples and Affymetrix Microarray Data on Matched Frozen Tissues. PLoS One 2016,11.

38. Zeng A, Yin J, Li Y, Li R, Wang Z, Zhou X, et al. miR-129-5p targets Wnt5a to block PKC/ERK/NF-KB and JNK pathways in glioblastoma. Cell Death Dis 9, 394 (2018).

39. Wang H, Wu B, Wang J, Hu Y, Dai X, Ye L, et al. Methylation associated miR-1246 contributes to poor prognosis in gliomas treated with temozolomide. Clin. Neurol. Neurosurg. 2021,200:106344.

40. Qiu G, Tong W, Jiang C, Xie Q, Zou J, Luo C, et al. Long Noncoding RNA WT1-AS Inhibit Cell Malignancy via miR-494-3p in Glioma. Technol. Cancer Res. Treat. 2020,19.

41. Jia Z, Wang K, Zhang A, Wang G, Kang C, Han L, et al. MiR-19a and miR-19b overexpression in gliomas. Pathol. Oncol. Res. 2013,19:847-53.

42. Xu G, Zhou H, Wang Q, Auersperg N, Peng C. Activin receptor-like kinase 7 induces apoptosis through up-regulation of Bax and down-regulation of Xiap in normal and malignant ovarian epithelial cell lines. Mol. Cancer Res. 2006,4:235-46.

43. Kwon SM, Kang SH, Park CK, Jung S, Park ES, Lee JS, et al. Recurrent glioblastomas reveal molecular subtypes associated with mechanistic implications of drug-resistance. PLoS One 2015,10.

44. Grin I, Ishchenko AA. An interplay of the base excision repair and mismatch repair pathways in active DNA demethylation. Nucleic Acids Res. 2016,44:3713-27.

45. Matsumoto T, Chino H, Akiya M, Hashimura M, Yokoi A, Tochimoto M, et al. Requirements of LEFTY and Nodal overexpression for tumor cell survival under hypoxia in glioblastoma. Mol. Carcinog. 2020,59:1409-19.

46. Yoshimatsu Y, Kimuro S, Pauty J, Takagaki K, Nomiyama S, Inagawa A, et al. TGF-beta and TNFalpha cooperatively induce mesenchymal transition of lymphatic endothelial cells via activation of Activin signals. PLoS One 2020,15.

47. Chang RX, Cui AL, Dong L, Guan SP, Jiang LY, Miao CX. Overexpression of RASAL1 indicates poor prognosis and promotes invasion of ovarian cancer. Open Life Sci. 2019,14:133-40.

48. Takashima Y, Kawaguchi A, Yamanaka R. Promising Prognosis Marker Candidates on the Status of Epithelial-Mesenchymal Transition and Glioma Stem Cells in Glioblastoma. Cells 2019,8. 
A

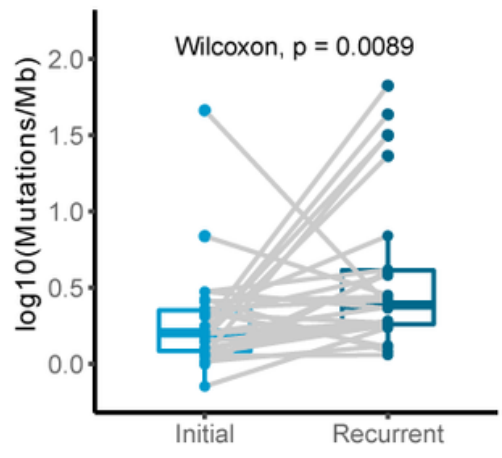

C

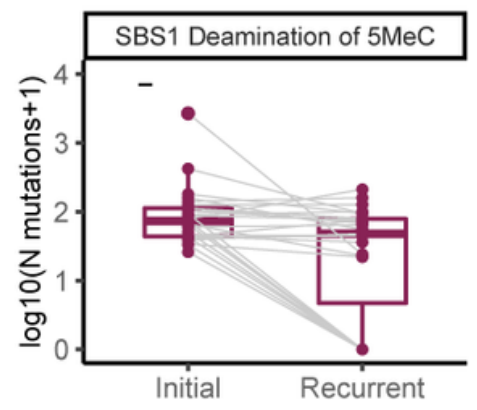

B

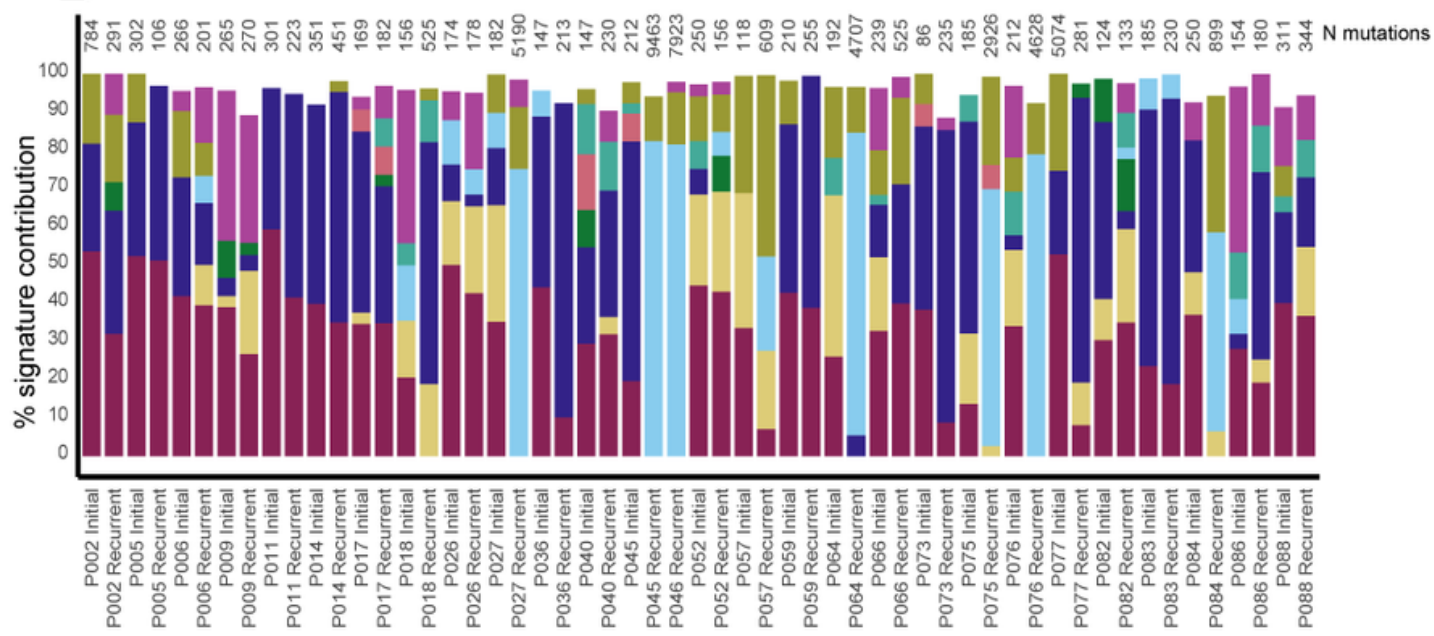

SBS1 Deamination of $5 \mathrm{MeC}$ SBS3 Defective HR repair

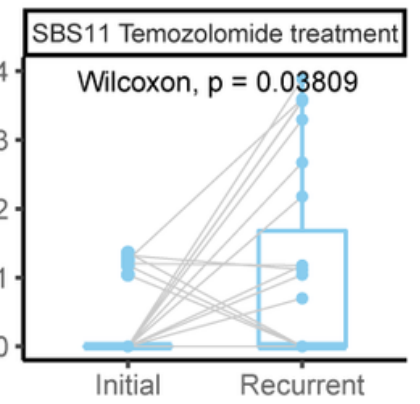

Figure 1

GBM recurrence is associated with increased Tumor mutational burden (TMB) and hypermutator phenotype. A) TMB in matched initial and recurrent tumor samples. B) Total number of mutations (synonymous \& non-synonymous, top) and \% contribution of selected COSMIC mutational signatures in each sample. C) Boxplots indicating the number of mutations contributing to the (left) SBS1 and (right) SBS11 signatures in the initial and recurrent samples. Lines join the paired tumor samples from a given patient. Signatures with no significant change are shown in suppl. Figure 4A. 


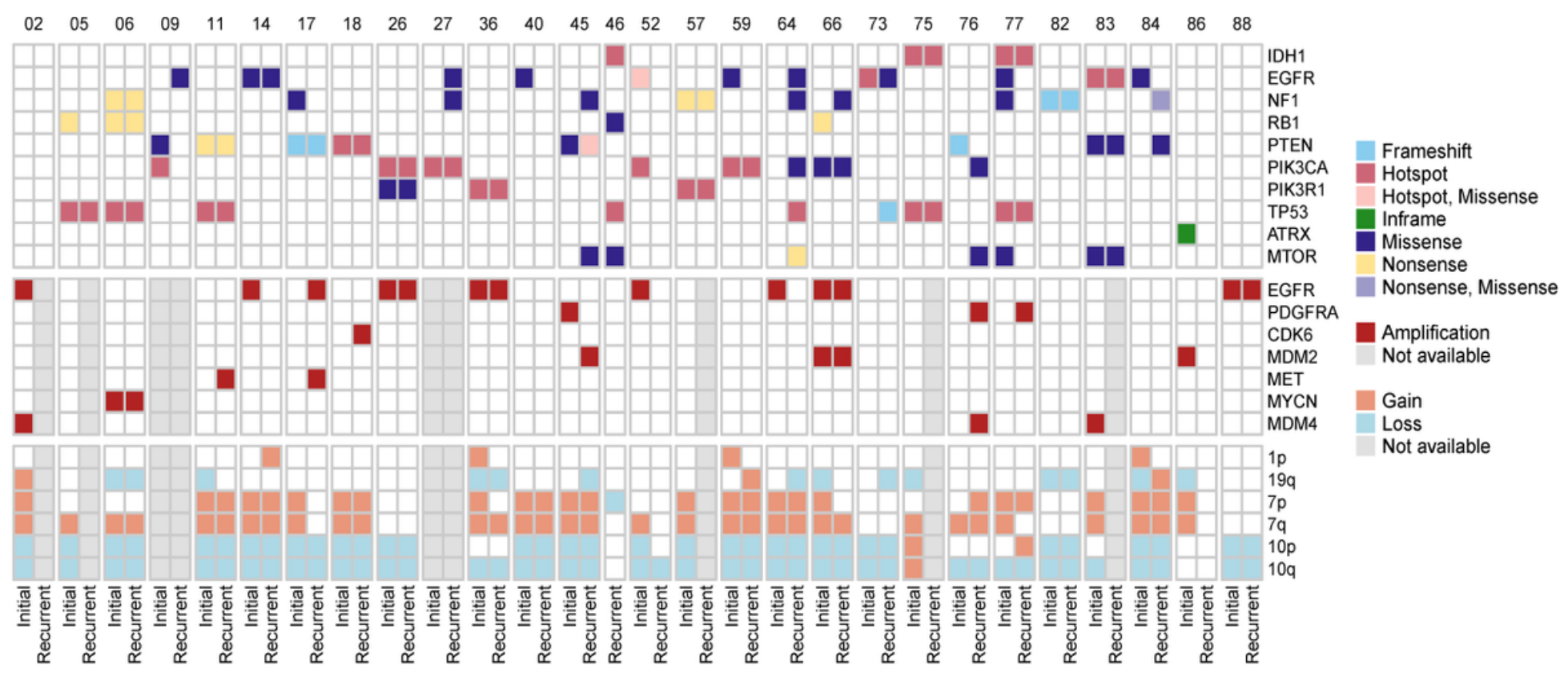

Figure 2

Mutational landscape of tumors with late recurrence. Heatmap depicting mutations (SNVs\&INDELs, top), amplifications (middle) and arm-level copy-number gains/losses (bottom) in GBM driver genes.

A

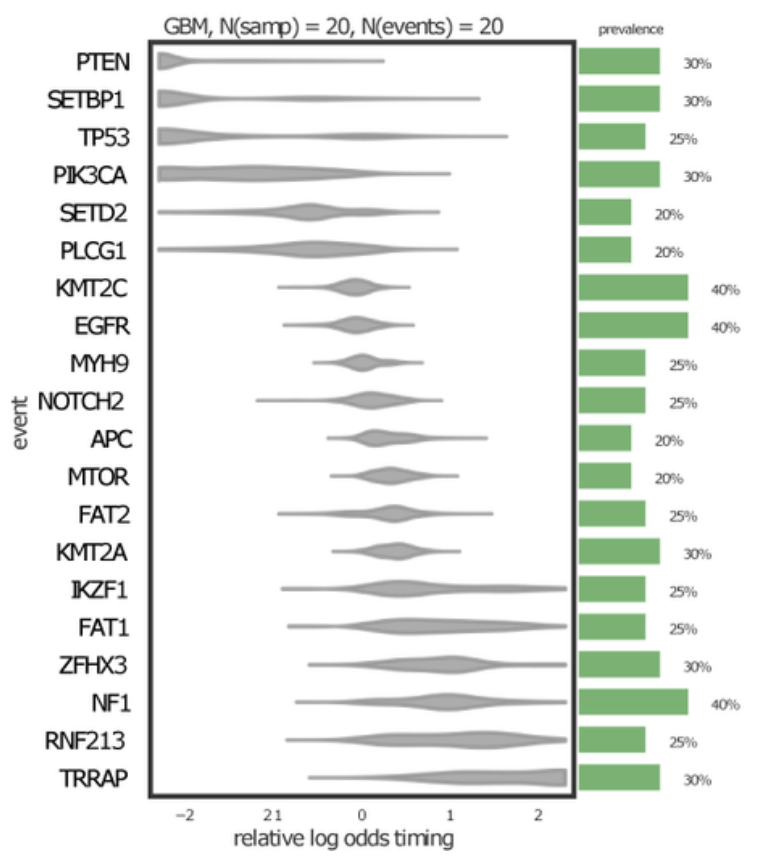

B
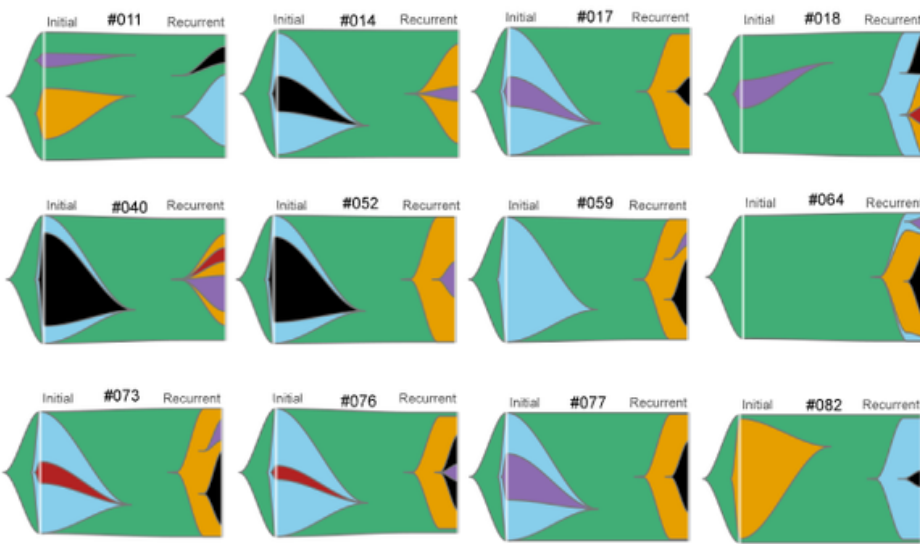

C
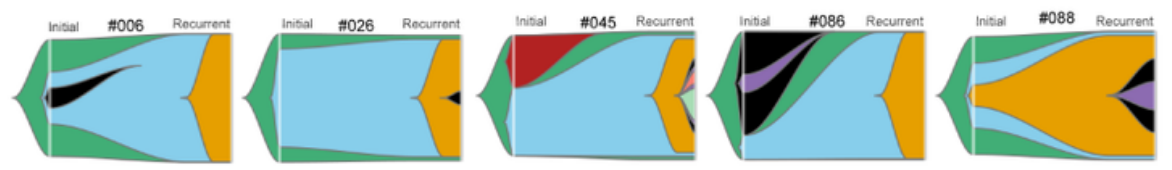

Figure 3

Acquistion of driver alterations over time in tumors with late recurrence. A) Estimated cohort-level timing of mutational events in 20 tumors with late recurrence. B) Fish plots showing tumors with early branching evolution (upper panel) and ongoing evolution of initial subclone (lower panel). 

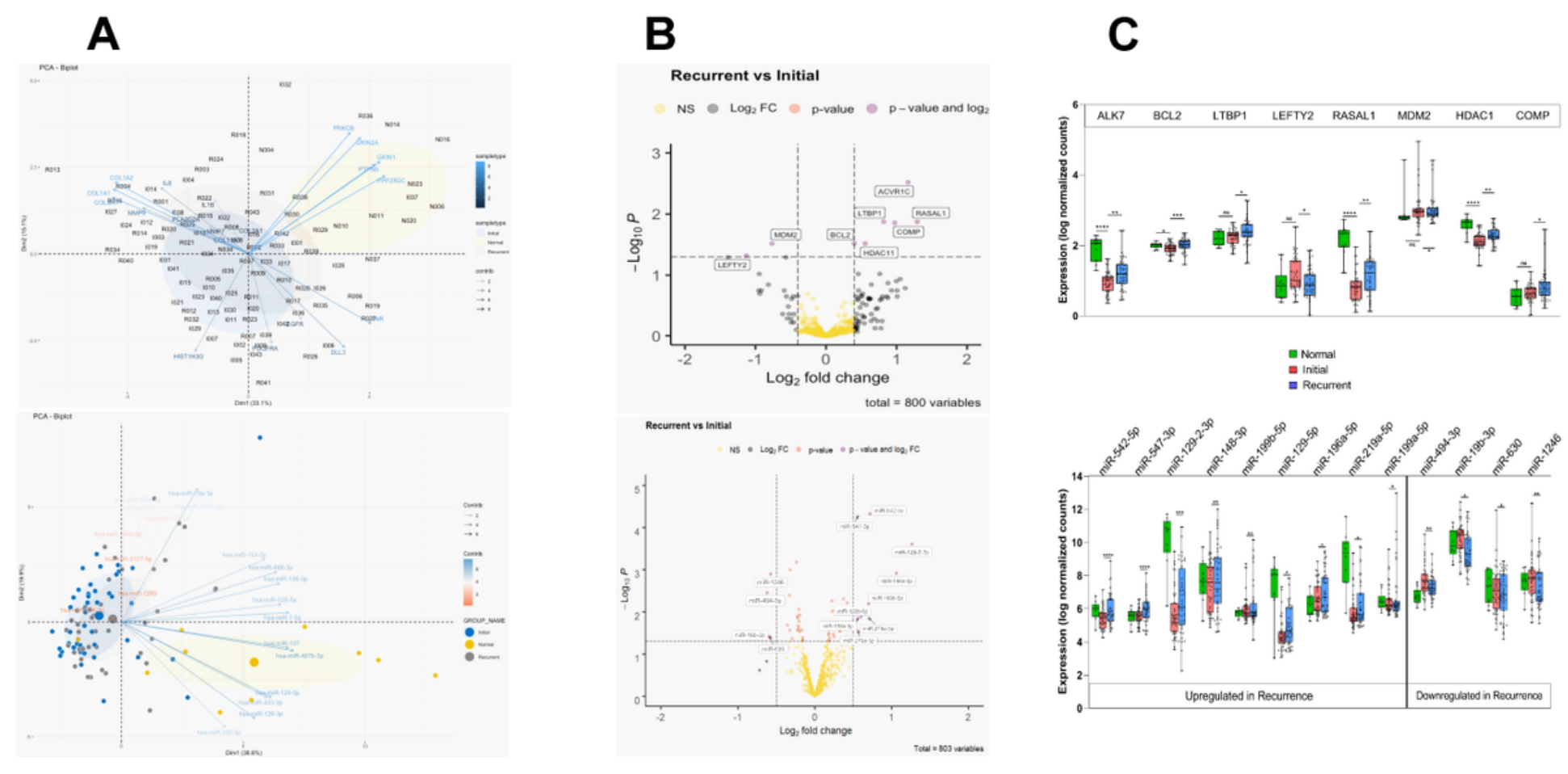

Figure 4

Differential pattern of mRNA and miRNA expression upon late recurrence. (A) Principal Component Analysis of mRNA (upper panel) and microRNA (lower panel) expression profiles. Note that the PCA plot from initial (I) and recurrent (R) samples almost completely overlap, but cluster separately from normal tissues (N). (B) Volcano plot indicating significantly up- (right) and down- (left) regulated mRNAs with significant adjusted p-value (upper panel) and miRNAs with significant p-value (lower panel). (C) Expression levels of significantly dysregulated mRNAs (upper panel) and miRNAs (lower panel) in primary, recurrent and adjacent normal tissues. ns $(P>0.05), *(P \leq 0.05), * \star(P \leq 0.01), * \star *(P \leq 0.001)$, $\star \star \star \star ~(P \leq 0.0001)$. 
A
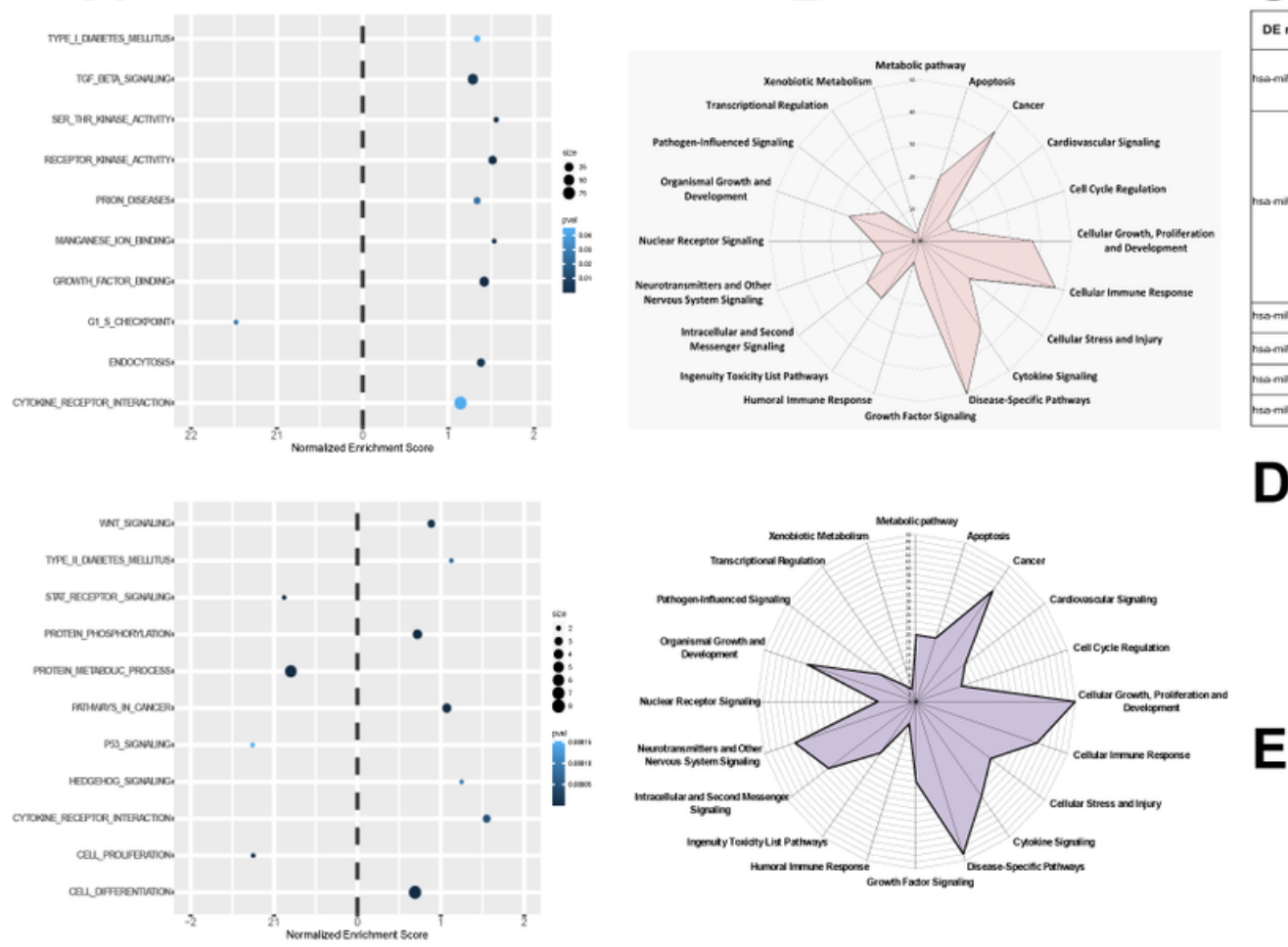

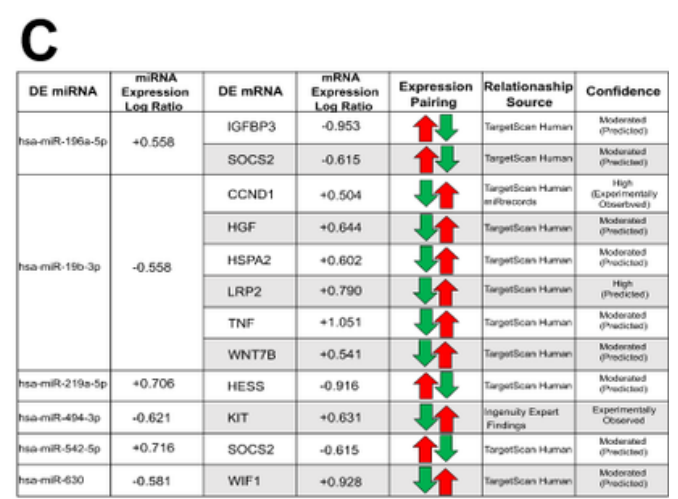

D

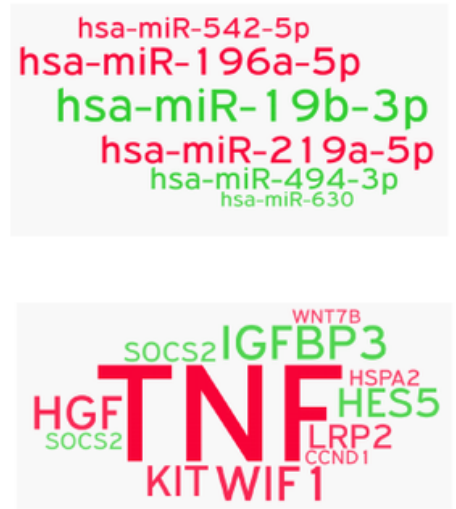

Figure 5

Deregulated pathways upon late recurrence (A) Gene set enrichment analysis of the mRNA (upper panel) and miRNA (lower panel) datasets for GO, KEGG and Reactome pathways. $X$ axis indicates the Normalized Enrichment Score (NES). Positive NES indicates upregulation and negative NES, downregulated on a given pathway in recurrence. Color indicates level of adjusted significance and dot size is proportional to the number of contributed leading edge genes. (B) Radar graph scored based on pathway analysis using IPA for mRNA data (upper panel) and miRNA data (lower panel). (C) Integration of mRNA and miRNA dataset and its translation into united deregulated pathways. Differentially regulated miRNAs with their inversely regulated putative mRNA targets. (D) Word cloud of differentially regulated miRNAs engaged in binding to their theoretical mRNA targets. (E) Word cloud of affected putative mRNA targets. Size implies number of targets, and level of impact by miR deregulation on putative targets. 

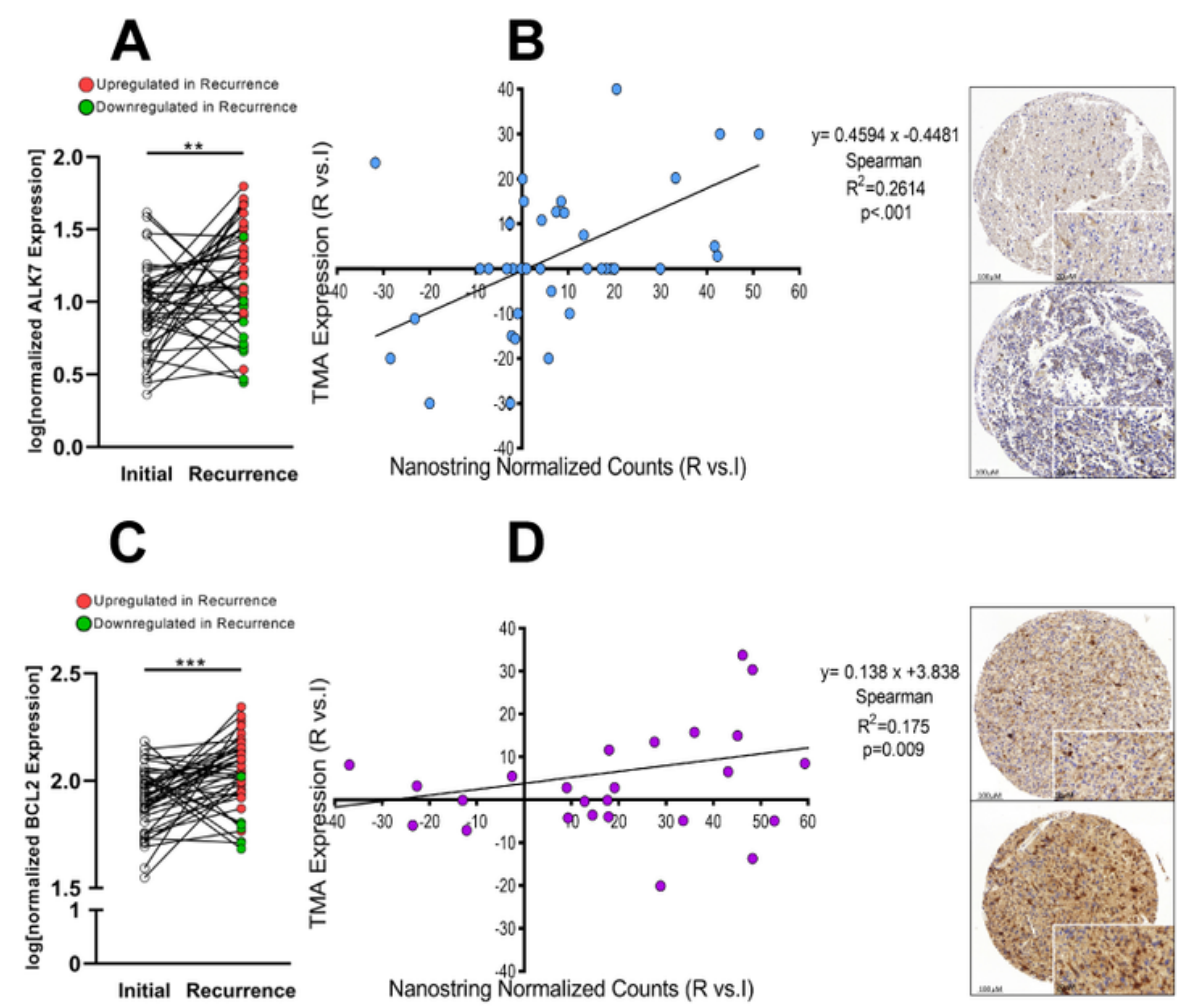

E
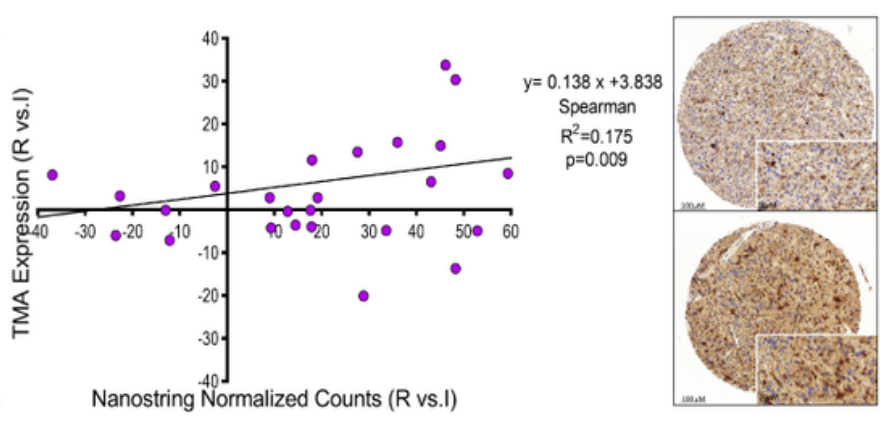

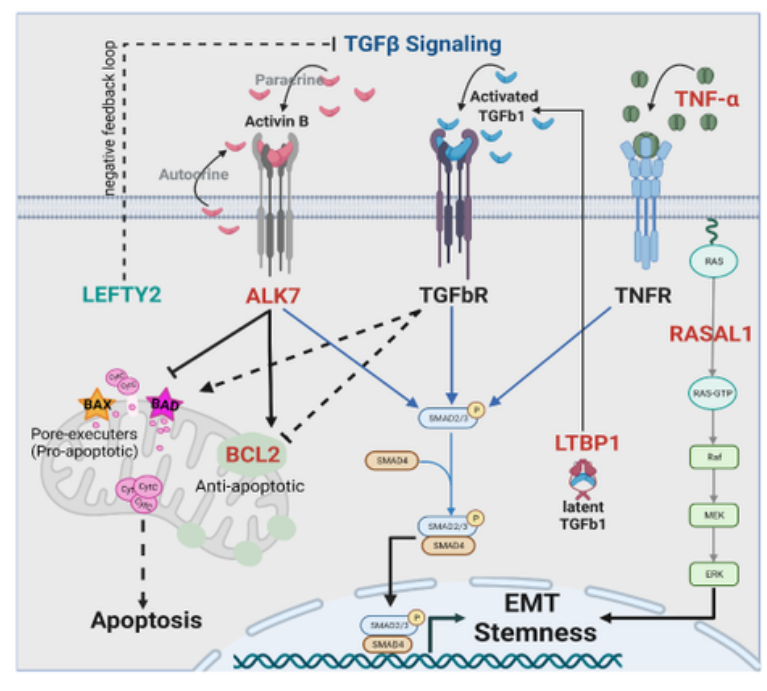

Figure 6

ALK7 is upregulated in the TMZ-resistant tumor of patients with late recurrence A) Pairwise comparison of ALK7 mRNA expression. B. Left: Correlation of tumor-specific ALK7 IHC scores and Nanostring normalized counts from tissue punches, Right: Example of TMA punches from the infiltration zone of initial (up) and center of recurrent (bottom) tumor from patient \#6 by ALK7 IHC. The upper panel shows neurons, which are ALK7 positive and tumor cells, which are ALK7 negative. C) Pairwise comparison of BCL2 mRNA expression. D) Correlation of BCL2 IHC scores and Nanostring normalized counts from tissue punches. Right: Example of TMA punches from the tumor center of initial (up) and recurrent (bottom) tumor from patient \#27 by BCL2 IHC. E) Model of concerted TGF $\beta$ signaling pathway. ALK7, LTBP1, RASAL1 upregulation and LEFTY2 downregulation translate into increased TGF $\beta$ induced EMT and stemness. To overcome TGF $\beta$-induced apoptosis, recurrent tumor cells downregulate pro-apoptotic proteins BAX and BAD and upregulate BCL-2 (see Fig. $4 A$ and suppl. Fig. 12). Dashed arrows indicate mechanisms that are uncoupled in recurrent tumors.

\section{Supplementary Files}

This is a list of supplementary files associated with this preprint. Click to download.

- SupplTable1.xlsx

- SupplTable2.xlsx

- SupplTable3.xlsx

- SuppITable4.xlsx 
- Suppl.FiguresMerged.pdf

- SupplementaryFigureLegends.docx

- SupplementaryMethods.docx 